\title{
Highly Efficient Addition of Activated Methylene Compounds to Alkenes Catalyzed by Gold and Silver
}

\author{
Xiaoquan Yao and Chao-Jun Li* \\ Department of chemistry, McGill University, 801 Sherbrooke St. West, Montreal, Quebec H3A 2K6, \\ Canada \\ Email:cj.li@,McGill.ca
}

\section{Supporting information}

All experiments were carried out under an inert atmosphere of nitrogen. Dichloromethane was refluxed and distilled from $\mathrm{CaH}_{2}$. Flash column chromatography was performed over SORBENT silica gel $30-60 \mu \mathrm{m} .{ }^{1} \mathrm{H}$ NMR and ${ }^{13} \mathrm{C}$ NMR spectra were acquired by Varian $400 \mathrm{MHz}$ and $100 \mathrm{MHz}$, or $300 \mathrm{MHz}$ and $75 \mathrm{MHz}$, respectively, and referenced to the internal solvent signals. IR spectra were recorded by ABB Bomem MB100 interferometer. MS data were obtained by KRATOS MS25RFA Mass Spectrometer. HRMS were made in McGill University.

Typical procedure (entry 1, Table 2): $\mathrm{AuCl}_{3}(15.2 \mathrm{mg}, 0.05 \mathrm{mmol})$ and $\mathrm{AgOTf}$ (38.5mg, $0.15 \mathrm{mmol}$ ) were added into $2 \mathrm{~mL}$ of dichloromethane under $\mathrm{N}_{2}$. The mixture was stirred at room temperature for 2 hours. 2,4-Pentanedione $(103 \mu \mathrm{L}, 1 \mathrm{mmol})$ was introduced into the solution of the catalyst prepared in situ. Styrene $(172 \mu \mathrm{L}, 1.5 \mathrm{mmol})$ was solved in $3 \mathrm{~mL}$ of $\mathrm{CH}_{2} \mathrm{Cl}_{2}$ and dropped into the mixture of reaction by syringe pump in 5 hours. The mixture was stirred at room temperature overnight and removed solvent under reduced pressure. The residuum was isolated by flash chromatography with silica to give diketone alkylation product with $89 \%$ yield. 


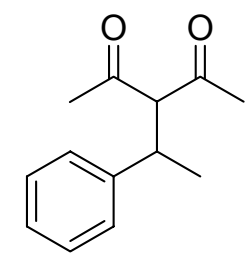

3-(1-phenylethyl)pentane-2,4-dione. CA registry Number: [5186-08-3]. IR (liquid film): $v_{\max } 3027,2964,1723,1700,1602,1494,1356,1185,1154,955,761,700 \mathrm{~cm}^{-1} ;{ }^{1} \mathrm{H}$ NMR $\left(\mathrm{CDCl}_{3}, 400 \mathrm{MHz}, \mathrm{ppm}\right) \delta 7.29-7.16(\mathrm{~m}, 5 \mathrm{H}), 4.05(\mathrm{~d}, J=11.2 \mathrm{~Hz}, 1 \mathrm{H}), 3.60(\mathrm{dq}, J=11.2$, $7.2 \mathrm{~Hz}, 1 \mathrm{H}), 2.26(\mathrm{~s}, 3 \mathrm{H}), 1.83(\mathrm{~s}, 3 \mathrm{H}), 1.21(\mathrm{~d}, J=7.2 \mathrm{~Hz}, 3 \mathrm{H}) ;{ }^{13} \mathrm{C} \mathrm{NMR}\left(\mathrm{CDCl}_{3}\right.$, $100 \mathrm{MHz}, \mathrm{ppm}) \delta 203.46,203.40,143.18,129.00,127.46,127.17,76.93,40.83,30.26$, $30.18,21.32$.

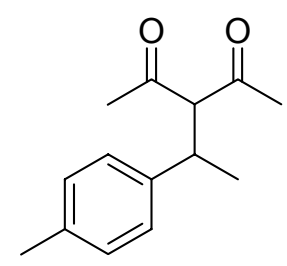

3-(1-p-tolylethyl)pentane-2,4-dione. IR (liquid film): $v_{\max }$ 3003, 2962, 2923, 1723, $1698,1514,1418,1356,1186,1155,955,816 \mathrm{~cm}^{-1} ;{ }^{1} \mathrm{H}$ NMR $\left(\mathrm{CDCl}_{3}, 300 \mathrm{MHz}, \mathrm{ppm}\right) \delta$ 7.12-7.08(m, 4H), 4.02(d, $J=11.7 \mathrm{~Hz}, 1 \mathrm{H}), 3.57(\mathrm{dq}, J=11.7,6.9 \mathrm{~Hz}, 1 \mathrm{H}), 2.31(\mathrm{~s}, 3 \mathrm{H})$, $2.28(\mathrm{~s}, 3 \mathrm{H}), 1.86(\mathrm{~s}, 3 \mathrm{H}), 1.20(\mathrm{~d}, J=6.9 \mathrm{~Hz}, 3 \mathrm{H}) ;{ }^{13} \mathrm{C} \mathrm{NMR}\left(\mathrm{CDCl}_{3}, 75 \mathrm{MHz}, \mathrm{ppm}\right) \delta$ $203.68,140.15,136.72,129.67,127.31,77.13,40.44,30.13,30.09,21.39,21.38$; MS (EI) $m / z(\%) 218\left(\mathrm{M}^{+}\right), 200,175(100), 161,157,142,119,91,77$; HRMS calcd for $\mathrm{C}_{14} \mathrm{H}_{18} \mathrm{O}_{2}: 218.1306$; found: 218.1296 .<smiles>COc1ccc(C(C)C(C(C)=O)C(C)=O)cc1</smiles> 
3-(1-(4-methoxyphenyl)ethyl)pentane-2,4-dione. IR (KBr): $v_{\max }$ 3008, 2965, 1719, $1696,1518,1363,1291,1251,1182,1037,838,830 \mathrm{~cm}^{-1} ;{ }^{1} \mathrm{H}$ NMR $\left(\mathrm{CDCl}_{3}, 400 \mathrm{MHz}\right.$, ppm) $\delta 7.08(\mathrm{~d}, J=8.8 \mathrm{~Hz}, 2 \mathrm{H}), 6.81(\mathrm{~d}, J=8.8 \mathrm{~Hz}, 2 \mathrm{H}), 3.97(\mathrm{~d}, J=11.2 \mathrm{~Hz}, 1 \mathrm{H}), 3.76(\mathrm{~s}$, $3 \mathrm{H}), 3.54(\mathrm{dq}, J=11.2,7.6 \mathrm{~Hz}, 1 \mathrm{H}), 2.26(\mathrm{~s}, 3 \mathrm{H}), 1.84(\mathrm{~s}, 3 \mathrm{H}), 1.18(\mathrm{~d}, J=7.6 \mathrm{~Hz}, 3 \mathrm{H}) ;{ }^{13} \mathrm{C}$ NMR $\left(\mathrm{CDCl}_{3}, 100 \mathrm{MHz}, \mathrm{ppm}\right) \delta 203.75,203.65,158.49,135.11,128.42,114.34,77.26$, 55.57, 40.14, 30.32, 30.14, 21.45; MS (EI) $m / z(\%)$ 234(M M $^{+}, 191,173,161,135(100)$, 120, 105, 91, 77; HRMS calcd for $\mathrm{C}_{14} \mathrm{H}_{18} \mathrm{O}_{3}: 234.1256$; found: 234.1251 .<smiles>CC(=O)C(C(C)=O)C(C)c1ccc(Cl)cc1</smiles>

3-(1-(4-chlorophenyl)ethyl)pentane-2,4-dione. IR (KBr): $v_{\max }$ 3002, 2977, 2966, 1724, $1699,1495,1363,1288,1146,1013,825,538 \mathrm{~cm}^{-1} ;{ }^{1} \mathrm{H} \mathrm{NMR}\left(\mathrm{CDCl}_{3}, 300 \mathrm{MHz}, \mathrm{ppm}\right) \delta$ $7.26(\mathrm{~d}, J=8.4 \mathrm{~Hz}, 2 \mathrm{H}), 7.12(\mathrm{~d}, J=8.4 \mathrm{~Hz}, 2 \mathrm{H}), 3.99(\mathrm{~d}, J=11.1 \mathrm{~Hz}, 1 \mathrm{H}), 3.59(\mathrm{dq}, J=$ $11.1,6.9 \mathrm{~Hz}, 1 \mathrm{H}), 2.27(\mathrm{~s}, 3 \mathrm{H}), 1.87(\mathrm{~s}, 3 \mathrm{H}), 1.19(\mathrm{~d}, J=6.9 \mathrm{~Hz}, 3 \mathrm{H}) ;{ }^{13} \mathrm{C} \mathrm{NMR}\left(\mathrm{CDCl}_{3}\right.$, 75MHz, ppm) $\delta 203.16,203.08,141.73,132.88,129.17,128.85,76.92,40.04,30.23$, 30.03, 21.17; MS (EI) $m / z(\%)$ 238(M $\left.\mathrm{M}^{+}\right), 220,195(100), 181,139,103,101,77$; HRMS calcd for $\mathrm{C}_{13} \mathrm{H}_{15} \mathrm{ClO}_{2}: 238.0761$; found: 238.0754 .<smiles>CC(=O)C(C(C)=O)C(C)c1ccccc1Cl</smiles> 
3-(1-(2-chlorophenyl)ethyl)pentane-2,4-dione. IR (KBr): $v_{\max }$ 3071, 2974, 1695, 1356, $1167,1035,756,689 \mathrm{~cm}^{-1} ;{ }^{1} \mathrm{H} \mathrm{NMR}\left(\mathrm{CDCl}_{3}, 400 \mathrm{MHz}, \mathrm{ppm}\right) \delta 7.35(\mathrm{~d}, J=8.0 \mathrm{~Hz}, 1 \mathrm{H})$, 7.21-7.13(m, 3H), 4.20(m, $2 \mathrm{H}), 2.27(\mathrm{~s}, 3 \mathrm{H}), 1.92(\mathrm{~s}, 3 \mathrm{H}), 1.17(\mathrm{~m}, 3 \mathrm{H}) ;{ }^{13} \mathrm{C} \mathrm{NMR}\left(\mathrm{CDCl}_{3}\right.$, $100 \mathrm{MHz}$, ppm) $\delta 203.17,203.10,140.53,133.55,130.37,128.23,128.04,127.56,75.38$, 36.24, 31.06, 28.58, 20.26; MS (EI) $\mathrm{m} / \mathrm{z}(\%)$ 238(M $\left.\mathrm{M}^{+}\right), 203,195(100), 181,161,159,139$, 125, 103, 101; HRMS calcd for $\mathrm{C}_{11} \mathrm{H}_{12} \mathrm{ClO}\left(\mathrm{M}^{+}-\mathrm{Ac}\right): 195.0577$; found: 195.0570 .

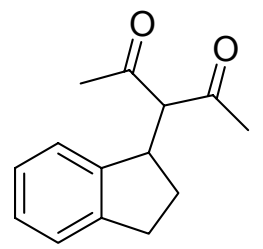

3-(2,3-dihydro-1H-inden-3-yl)pentane-2,4-dione. IR (liquid film): $v_{\max }$ 3068, 3020, 2944, 2848, 1724, 1697, 1457, 1357, 1256, 1156, 1023, 952, $750 \mathrm{~cm}^{-1} ;{ }^{1} \mathrm{H} \mathrm{NMR}\left(\mathrm{CDCl}_{3}\right.$, $300 \mathrm{MHz}, \mathrm{ppm}) \delta 7.25-7.00(\mathrm{~m}, 4 \mathrm{H}), 4.02(\mathrm{~m}, 1 \mathrm{H}), 3.89(\mathrm{~d}, J=10.2 \mathrm{~Hz}, 1 \mathrm{H}), 3.10-2.80(\mathrm{~m}$, $2 \mathrm{H}), 2.34-2.22(\mathrm{~m}, 1 \mathrm{H}), 2.20(\mathrm{~s}, 3 \mathrm{H}), 2.17(\mathrm{~s}, 3 \mathrm{H}), 1.70(\mathrm{~m}, 1 \mathrm{H}), ;{ }^{13} \mathrm{C} \mathrm{NMR}\left(\mathrm{CDCl}_{3}\right.$, $100 \mathrm{MHz}$, ppm) $\delta 204.89,203.55,143.98,143.60,127.53,126.63,125.12,124.29,74.13$, 44.87, 31.54, 31.23, 30.42, 29.61; MS (EI) $m / z(\%)$ 216(M M $^{+}, 173(100), 155,129,116$, 101, 91, 43; HRMS calcd for $\mathrm{C}_{14} \mathrm{H}_{16} \mathrm{O}_{2}: 216.1150$; found: 216.1155 .

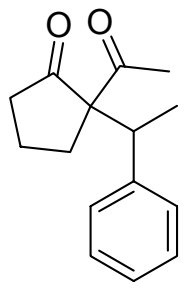

2-acetyl-2-(1-phenylethyl)cy-clopentanone. (The two diastereomers are difficult to be separated by flash column chromatography. But one of them can be isolated from the 
mixture partly by crystallization) One of the two isomers(pure): $\mathrm{IR}(\mathrm{KBr}): v_{\max } 3066$, $3038,2967,2953,1734,1698,1497,1360,1143,1122,829,763,706 \mathrm{~cm}^{-1} ;{ }^{1} \mathrm{H}$ NMR $\left(\mathrm{CDCl}_{3}, 400 \mathrm{MHz}, \mathrm{ppm}\right) \delta 7.26-7.12(\mathrm{~m}, 5 \mathrm{H}), 3.79(\mathrm{q}, J=6.8 \mathrm{~Hz}, 1 \mathrm{H}), 2.76-2.71(\mathrm{~m}, 1 \mathrm{H})$, 2.39-2.31(m, 1H), 2.17-2.07(m, 2H), 2.09(s, 3H), 1.88-1.70(m, 1H), 1.78-1.68(m, 1H), $1.21(\mathrm{~d}, J=6.8 \mathrm{~Hz}, 3 \mathrm{H}) ;{ }^{13} \mathrm{C} \mathrm{NMR}\left(\mathrm{CDCl}_{3}, 100 \mathrm{MHz}, \mathrm{ppm}\right) \delta 215.99,202.98,142.32$, $128.81,127.96,127.21,74.83,44.51,39.81,27.57,26.57,20.00,19.24$; MS (EI) $m / z(\%)$ 230( $\left.\mathrm{M}^{+}\right), 212,188,187,173,159,126,105(100), 91,77$; HRMS calcd for $\mathrm{C}_{15} \mathrm{H}_{18} \mathrm{O}_{2}$ : 230.1307; found: 230.1299 .

Another diastereomer (from the mixture of two isomers, and some peaks are difficult to be distinguished): ${ }^{1} \mathrm{H} \mathrm{NMR}\left(\mathrm{CDCl}_{3}, 400 \mathrm{MHz}, \mathrm{ppm}\right) \delta 3.94(\mathrm{q}, J=7.2 \mathrm{~Hz}, 1 \mathrm{H}), 2.39(\mathrm{~s}$, $3 \mathrm{H}), 1.17(\mathrm{~d}, J=7.2 \mathrm{~Hz}, 3 \mathrm{H}) ;{ }^{13} \mathrm{C} \mathrm{NMR}\left(\mathrm{CDCl}_{3}, 100 \mathrm{MHz}, \mathrm{ppm}\right) \delta 215.45,203.23,140.88$, $75.16,43.28,40.25,25.96,25.05,19.81,16.50$.

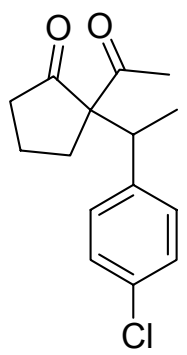

2-acetyl-2-(1-(4-chlorophenyl)ethyl)cyclopentanone. IR (KBr): $v_{\max }$ 3038, 2973, 1735, 1700, 1493, 1355, 1196, 1143, 1094, 1014, 835, $666 \mathrm{~cm}^{-1} ;{ }^{1} \mathrm{H}$ NMR $\left(\mathrm{CDCl}_{3}, 400 \mathrm{MHz}\right.$, ppm) $\delta 7.24(\mathrm{~d}, J=8.0 \mathrm{~Hz}, 2 \mathrm{H}), 7.13(\mathrm{~d}, J=8.0 \mathrm{~Hz}, 2 \mathrm{H}), 3.90(\mathrm{q}, J=6.8 \mathrm{~Hz}, 1 \mathrm{H}), 2.72-$ $2.66(\mathrm{~m}, 1 \mathrm{H}), 2.37(\mathrm{~s}, 3 \mathrm{H}), 2.08-1.40(\mathrm{~m}, 5 \mathrm{H}), 1.14(\mathrm{~d}, J=6.8 \mathrm{~Hz}, 3 \mathrm{H}) ;{ }^{13} \mathrm{C} \mathrm{NMR}(\mathrm{CDCl} 3$, $100 \mathrm{MHz}, \mathrm{ppm}) \delta 215.32,202.81,139.45,133.14,130.71,128.52,75.10,42.50,40.11$, 
25.81, 25.05, 19.73, 16.45; MS (EI) $m / z(\%) 264\left(\mathrm{M}^{+}\right), 221(100), 207,141,139,115,103$, 77; HRMS calcd for $\mathrm{C}_{15} \mathrm{H}_{17} \mathrm{ClO}_{2}: 264.0917$, found: 264.0913 .

Another diastereomer: IR (KBr): $v_{\max } 3034,2966,1736,1696,1500,1489,1359,1147$, $1121,1014,840,820 \mathrm{~cm}^{-1} ;{ }^{1} \mathrm{H} \mathrm{NMR}\left(\mathrm{CDCl}_{3}, 300 \mathrm{MHz}, \mathrm{ppm}\right) \delta 7.22(\mathrm{~d}, J=8.4 \mathrm{~Hz}, 2 \mathrm{H})$, $7.06(\mathrm{~d}, J=8.4 \mathrm{~Hz}, 2 \mathrm{H}), 3.77(\mathrm{q}, J=7.2 \mathrm{~Hz}, 1 \mathrm{H}), 2.75-2.68(\mathrm{~m}, 1 \mathrm{H}), 2.42-2.30(\mathrm{~m}, 1 \mathrm{H})$, $2.20-2.06(\mathrm{~m}, 2 \mathrm{H}), 2.09(\mathrm{~s}, 3 \mathrm{H}), 1.95-1.64(\mathrm{~m}, 2 \mathrm{H}), 1.19(\mathrm{~d}, J=7.2 \mathrm{~Hz}, 3 \mathrm{H}) ;{ }^{13} \mathrm{C} \mathrm{NMR}$ $\left(\mathrm{CDCl}_{3}, 75 \mathrm{MHz}, \mathrm{ppm}\right) \delta 215.70,202.71,140.87,133.03,129.33,128.99,74.59,43.83$, 39.67, 27.43, 26.50, 19.85, 19.07; MS (EI) $\mathrm{m} / z(\%) 264\left(\mathrm{M}^{+}\right), 221(100), 207,141,139$, 115, 103, 77; HRMS calcd for $\mathrm{C}_{15} \mathrm{H}_{17} \mathrm{ClO}_{2}: 264.0917$, found: 264.0910 .

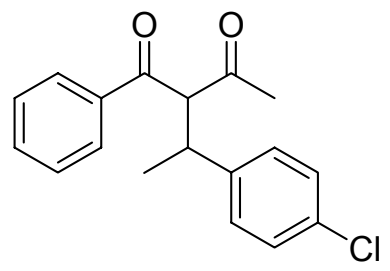

2-(1-(4-chlorophenyl)ethyl)-1-phenylbutane-1,3-dione. IR (KBr): $v_{\max }$ 3068, 2971, $1711,1675,1594,1355,1265,1218,1014,971,826,767 \mathrm{~cm}^{-1} ;{ }^{1} \mathrm{H}$ NMR (CDCl3, $400 \mathrm{MHz}, \mathrm{ppm}) \delta 8.07(\mathrm{~m}, 2 \mathrm{H}), 7.64-7.48(\mathrm{~m}, 3 \mathrm{H}), 7.30-7.20(\mathrm{~m}, 4 \mathrm{H}), 4.86(\mathrm{~d}, \mathrm{~J}=10.8 \mathrm{~Hz}$, $1 \mathrm{H}), 3.87(\mathrm{dq}, J=10.8,7.2 \mathrm{~Hz}, 1 \mathrm{H}), 1.92(\mathrm{~s}, 3 \mathrm{H}), 1.20(\mathrm{~d}, J=7.2 \mathrm{~Hz}, 3 \mathrm{H}) ;{ }^{13} \mathrm{C}$ NMR $\left(\mathrm{CDCl}_{3}, 100 \mathrm{MHz}, \mathrm{ppm}\right) \delta 202.90,194.94,141.87,137.21,134.18,132.90,129.18$, 129.14, 129.09, 71.01, 40.61, 28.37, 21.91; MS (EI) $\mathrm{m} / z(\%)$ 300(M $\left.\mathrm{M}^{+}\right), 257(100), 243$, 195, 179, 139, 105, 77; HRMS calcd for $\mathrm{C}_{18} \mathrm{H}_{17} \mathrm{ClO}_{2}: 300.0917$, found: 300.0910 . Another diastereomer: IR (KBr): $v_{\max } 3066,2972,1713,1670,1596,1494,1360,1218$, $1176,1098,1012,836,759 \mathrm{~cm}^{-1} ;{ }^{1} \mathrm{H} \mathrm{NMR}\left(\mathrm{CDCl}_{3}, 400 \mathrm{MHz}, \mathrm{ppm}\right) \delta 7.81-7.78(\mathrm{~m}, 2 \mathrm{H})$, 7.51(m, 1H), 7.40-7.36(m, 2H), 7.13(s, $4 \mathrm{H}), 4.77(\mathrm{~d}, J=10.8 \mathrm{~Hz}, 1 \mathrm{H}), 3.84(\mathrm{dq}, J=10.8$ 
$7.2 \mathrm{~Hz}, 1 \mathrm{H}), 2.24(\mathrm{~s}, 3 \mathrm{H}), 1.29(\mathrm{~d}, J=7.2 \mathrm{~Hz}, 3 \mathrm{H}) ;{ }^{13} \mathrm{C} \mathrm{NMR}\left(\mathrm{CDCl}_{3}, 100 \mathrm{MHz}, \mathrm{ppm}\right) \delta$ 203.37, 195.00, 142.18, 136.96, 133.88, 132.49, 128.96, 128.93, 128.81, 128.69, 71.73, 39.96, 27.90, 20.62; MS (EI) $m / z(\%) 300\left(\mathrm{M}^{+}\right), 257(100), 243,195,179,139,105,77$; HRMS calcd for $\mathrm{C}_{18} \mathrm{H}_{17} \mathrm{ClO}_{2}: 300.0917$, found: 300.0908 .

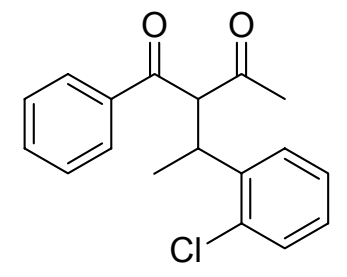

2-(1-(2-chlorophenyl)ethyl)-1-phenylbutane-1,3-dione. IR (KBr): $v_{\max }$ 3071, 2969, $1715,1665,1579,1479,1356,1264,1210,1172,1033,970,761,689 \mathrm{~cm}^{-1} ;{ }^{1} \mathrm{H}$ NMR $\left(\mathrm{CDCl}_{3}, 400 \mathrm{MHz}, \mathrm{ppm}\right) \delta 8.05(\mathrm{~d}, J=8.4 \mathrm{~Hz}, 2 \mathrm{H}), 7.59-7.14(\mathrm{~m}, 7 \mathrm{H}), 5.04(\mathrm{~d}, J=9.6 \mathrm{~Hz}$, $1 \mathrm{H}), 4.49(\mathrm{dq}, J=9.6,6.8 \mathrm{~Hz}, 1 \mathrm{H}), 1.97(\mathrm{~s}, 3 \mathrm{H}), 1.19(\mathrm{~d}, J=6.8 \mathrm{~Hz}, 3 \mathrm{H}) ;{ }^{13} \mathrm{C} \mathrm{NMR}\left(\mathrm{CDCl}_{3}\right.$, $75 \mathrm{MHz}$, ppm) $\delta 203.36,195.16,140.51,137.29,134.08,133.55,130.33,129.15,129.06$, 128.26, 127.54, 69.47, 36.99, 27.46, 20.45; MS (EI) $m / z(\%) 265\left(\mathrm{M}^{+}-\mathrm{Cl}\right), 257,243,223$, 195, 139, 105(100), 77; HRMS calcd for $\mathrm{C}_{18} \mathrm{H}_{17} \mathrm{O}_{2}\left(\mathrm{M}^{+}-\mathrm{Cl}\right)$ : 265.1229, found: 265.1225. Another diastereoisomer: IR (KBr): $v_{\max } 3057,2971,1713,1670,1595,1475,1436$, $1215,1173,1035,975,770,697 \mathrm{~cm}^{-1} ;{ }^{1} \mathrm{H}$ NMR $\left(\mathrm{CDCl}_{3}, 300 \mathrm{MHz}, \mathrm{ppm}\right) \delta 7.89(\mathrm{~d}, J=$ $7.5 \mathrm{~Hz}, 2 \mathrm{H}), 7.60-7.03(\mathrm{~m}, 7 \mathrm{H}), 5.05(\mathrm{~d}, J=10.5,1 \mathrm{H}), 4.36(\mathrm{dq}, J=10.5,7.2 \mathrm{~Hz}, 1 \mathrm{H})$, 2.26(s, 3H), $1.30(\mathrm{~d}, J=7.2 \mathrm{~Hz}, 3 \mathrm{H}) ;{ }^{13} \mathrm{C} \mathrm{NMR}\left(\mathrm{CDCl}_{3}, 75 \mathrm{MHz}, \mathrm{ppm}\right) \delta 203.69,194.87$, $141.12,136.87,133.81,130.28,128.94,128.75,127.89,127.22,69.74,36.71,28.23$, 19.62; MS (EI) $m / z(\%)$ 265(M+Cl), 257, 243, 223, 195, 139, 105(100), 77, HRMS calcd for $\mathrm{C}_{18} \mathrm{H}_{17} \mathrm{O}_{2}\left(\mathrm{M}^{+}-\mathrm{Cl}\right): 265.1229$, found: 265.1225 . 


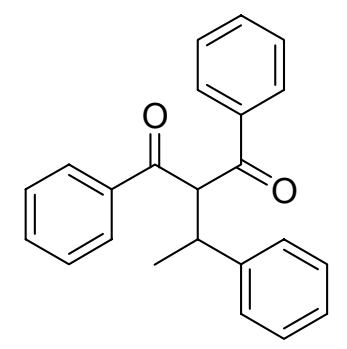

1,3-diphenyl-2-(1-phenylethyl)propane-1,3-dione. CA registry Number: [116140-580]. IR (KBr): $v_{\max } 3070,3001,2972,1687,1650,1594,1447,1275,1213,1213,1000$, $972,769 \mathrm{~cm}^{-1} ;{ }^{1} \mathrm{H} \mathrm{NMR}\left(\mathrm{CDCl}_{3}, 300 \mathrm{MHz}, \mathrm{ppm}\right) \delta 8.04(\mathrm{~d}, J=7.5 \mathrm{~Hz}, 2 \mathrm{H}), 7.74(\mathrm{~d}, J=$ $7.5 \mathrm{~Hz}, 2 \mathrm{H}), 7.60-7.07(\mathrm{~m}, 11 \mathrm{H}), 5.61(\mathrm{~d}, J=9.9 \mathrm{~Hz}, 1 \mathrm{H}), 4.09(\mathrm{dq}, J=9.9,6.9 \mathrm{~Hz}, 1 \mathrm{H})$, $1.36(\mathrm{~d}, J=6.9 \mathrm{~Hz}, 3 \mathrm{H}) ;{ }^{13} \mathrm{C} \mathrm{NMR}\left(\mathrm{CDCl}_{3}, 75 \mathrm{MHz}, \mathrm{ppm}\right) \delta 195.04,194.69,143.99$, $137.34,137.09,133.74,133.23,129.05,129.02,128.70,128.65,128.60,127.94,126.81$, 65.22, 41.53, 20.63; MS (EI) m/z (\%) 327(M+H), 259, 241, 223(100), 147, 129, $105,77$.

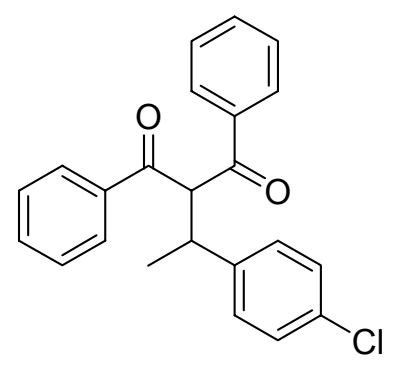

2-(1-(4-chlorophenyl)ethyl)-1,3-diphenylpropane-1,3-dione. IR (KBr): $v_{\max }$ 3057, $2971,1689,1663,1594,1577,1447,1284,1284,1099,970,762 \mathrm{~cm}^{-1} ;{ }^{1} \mathrm{H} \mathrm{NMR}\left(\mathrm{CDCl}_{3}\right.$, $400 \mathrm{MHz}, \mathrm{ppm}) \delta 8.04(\mathrm{~d}, J=8.0 \mathrm{~Hz}, 2 \mathrm{H}), 7.75(\mathrm{~d}, J=8.0 \mathrm{~Hz}, 2 \mathrm{H}), 7.58-7.12(\mathrm{~m}, 10 \mathrm{H})$, $5.57(\mathrm{~d}, J=10.4 \mathrm{~Hz}, 1 \mathrm{H}), 4.07(\mathrm{dq}, J=10.4,6.8 \mathrm{~Hz}, 1 \mathrm{H}), 1.32(\mathrm{~d}, J=6.8 \mathrm{~Hz}, 3 \mathrm{H}) ;{ }^{13} \mathrm{C} \mathrm{NMR}$ $\left(\mathrm{CDCl}_{3}, 100 \mathrm{MHz}, \mathrm{ppm}\right) \delta 194.74,194.39,142.52,137.10,136.83,133.90,133.49$, $132.43,129.32,129.13,129.02,128.78,128.71,128.69,65.03,40.96,20.74 ; \mathrm{MS}(\mathrm{EI}) \mathrm{m} / \mathrm{z}$ 
(\%) 362( $\left.\mathrm{M}^{+}-\mathrm{H}\right), 327,257(100), 223,147,139,105,77$; HRMS calcd for $\mathrm{C}_{16} \mathrm{H}_{14} \mathrm{ClO}\left(\mathrm{M}^{+}-\right.$ COPh): 257.0733, found: 257.0731 .<smiles>CC(c1ccccc1Cl)C(C(=O)c1ccccc1)C(=O)c1ccccc1</smiles>

2-(1-(2-chlorophenyl)ethyl)-1,3-diphenylpropane-1,3-dione. IR (KBr): $v_{\max }$ 3064, $2984,1695,1657,1594,1447,1290,1198,1035,988,751,688 \mathrm{~cm}^{-1} ;{ }^{1} \mathrm{H} \mathrm{NMR}\left(\mathrm{CDCl}_{3}\right.$, $400 \mathrm{MHz}, \mathrm{ppm}) \delta 7.95(\mathrm{~m}, 2 \mathrm{H}), 7.86(\mathrm{~m}, 2 \mathrm{H}), 7.54-7.22(\mathrm{~m}, 8 \mathrm{H}), 7.07-7.04(\mathrm{~m}, 2 \mathrm{H}), 5.87(\mathrm{~d}$, $J=8.4 \mathrm{~Hz}, 1 \mathrm{H}), 4.48(\mathrm{dq}, J=8.4,7.2 \mathrm{~Hz}, 1 \mathrm{H}), 1.37(\mathrm{~d}, J=7.2 \mathrm{~Hz}, 3 \mathrm{H}) ;{ }^{13} \mathrm{C} \mathrm{NMR}\left(\mathrm{CDCl}_{3}\right.$, $100 \mathrm{MHz}, \mathrm{ppm}) \delta 195.42,194.66,141.24,137.17,136.69,133.76,133.72,133.40$, $130.15,129.01,128.79,128.68,127.93,127.05,62.00,32.03,18.69 ; \mathrm{MS}(\mathrm{CI}) \mathrm{m} / z(\%)$ $363\left(\mathrm{M}^{+}+\mathrm{H}\right), 327,257,225,207,139,105(100), 77$; HRMS calcd for $\mathrm{C}_{23} \mathrm{H}_{19} \mathrm{O}_{2}\left(\mathrm{M}^{+}-\mathrm{Cl}\right)$ : 327.1385, found: 327.1380 .<smiles>CCC(c1ccccc1)C(C(=O)c1ccccc1)C(=O)c1ccccc1</smiles>

1,3-diphenyl-2-(1-phenylpropyl)propane-1,3-dione. IR (KBr): $v_{\max }$ 3060, 2979, 1685, 1661, 1594, 1447, 1277, 1196, 986, 963, 759, $701 \mathrm{~cm}^{-1} ;{ }^{1} \mathrm{H}$ NMR $\left(\mathrm{CDCl}_{3}, 400 \mathrm{MHz}\right.$, ppm) $\delta 8.07(\mathrm{~d}, J=6.8 \mathrm{~Hz}, 2 \mathrm{H}), 7.72(\mathrm{~d}, J=7.2 \mathrm{~Hz}, 2 \mathrm{H}), 7.56-7.06(\mathrm{~m}, 11 \mathrm{H}), 5.69(\mathrm{~d}, J=$ $10.0,1 \mathrm{H}), 3.84(\mathrm{~m}, 1 \mathrm{H}), 1.79-1.66(\mathrm{~m}, 2 \mathrm{H}), 0.72(\mathrm{dd}, J=7.2,7.4 \mathrm{~Hz}, 3 \mathrm{H}) ;{ }^{13} \mathrm{C}$ NMR 
$\left(\mathrm{CDCl}_{3}, 100 \mathrm{MHz}, \mathrm{ppm}\right) \delta 195.13,194.44,141.35,137.40,137.16,133.79,133.18$, $129.08,129.03,128.87,128.69,128.60,128.46,126.79,64.74,49.16,27.47,12.54 ; \mathrm{MS}$ (EI) $m / z(\%) 342\left(\mathrm{M}^{+}\right), 313,257,237(100), 223,195,147,129,105,77$; HRMS calcd for $\mathrm{C}_{24} \mathrm{H}_{22} \mathrm{O}_{2}: 342.1620$, found: 342.1607 .

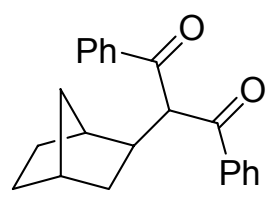

2-(bicyclo[2.2.1]heptan-2-yl)-1,3-diphenylpropane-1,3-dione. IR (KBr): $v_{\max }$ 3068, 2652, 2906, 2861, 1694, 1656, 1448, 1287, 1200, 985, 758, 699 $\mathrm{cm}^{-1} ;{ }^{1} \mathrm{H}$ NMR $\left(\mathrm{CDCl}_{3}\right.$, $400 \mathrm{MHz}, \mathrm{ppm}) \delta 8.03(\mathrm{~d}, J=7.2 \mathrm{~Hz}, 2 \mathrm{H}), 7.93(\mathrm{~d}, J=6.8 \mathrm{~Hz}, 2 \mathrm{H}), 7.57-7.37(\mathrm{~m}, 6 \mathrm{H})$, 5.03(d, $J=10.8,1 \mathrm{H}), 2.74(\mathrm{~m}, 1 \mathrm{H}), 2.24(\mathrm{~s}, 1 \mathrm{H}), 1.96(\mathrm{~s}, 1 \mathrm{H}), 1.62(\mathrm{~m}, 1 \mathrm{H}), 1.51-1.48(\mathrm{~m}$, $3 \mathrm{H}), 1.30(\mathrm{~m}, 1 \mathrm{H}), 1.22-1.19(\mathrm{~m}, 2 \mathrm{H}), 1.13-1.10(\mathrm{~m}, 1 \mathrm{H}) ;{ }^{13} \mathrm{C} \mathrm{NMR}\left(\mathrm{CDCl}_{3}, 100 \mathrm{MHz}\right.$, ppm) $\delta 195.87,195.26,137.34,137.25,133.66,133.45,129.07,129.05,128.93,128.89$, 64.73, 44.03, 40.02, 37.63, 37.11, 36.43, 30.46, 28.94; MS (EI) $\mathrm{m} / z(\%) 318\left(\mathrm{M}^{+}\right), 300$, 224, 213, 196, 167, 143, 105, 77; HRMS calcd for $\mathrm{C}_{22} \mathrm{H}_{22} \mathrm{O}_{2}: 318.1620$, found: 318.1614 . The configuration of the product was determined by NOE. 